\title{
Modified uniportal video-assisted thoracoscopic surgery (VATS)
}

\author{
H. Volkan Kara ${ }^{1}$, Stafford S. Balderson ${ }^{2}$, Thomas A. D'Amico ${ }^{2}$ \\ ${ }^{1}$ Department of Thoracic Surgery, Istanbul University, Cerrahpasa Medical Faculty Istanbul, Turkey; ${ }^{2}$ Department of Surgery, Division of Thoracic \\ Surgery, Duke University Medical Center, Durham, NC, USA \\ Correspondence to: H. Volkan Kara, MD, MSc. Department of Thoracic Surgery Istanbul University Cerrahpasa Medical Faculty, Fatih 34098 \\ Istanbul, Turkey. Email: volkan_kara@yahoo.com.
}

\begin{abstract}
Video-assisted thoracoscopic surgery (VATS) for resectable lung cancer patients has been frequently used in the past decades. The potential beneficial advantages and safety of VATS has been shown in large patient series and meta-analyses. The strategy of limiting access to one incision in one intercostal space (uniportal VATS) has been adopted by some thoracic surgeons in recent years. We have described a modified uniportal VATS technique with its potential advantages. Modified uniportal VATS potentially offers better exposure, beneficial opportunities for education and improved comfort for the thoracic surgery team in clinical usage.
\end{abstract}

Keywords: Video-assisted thoracoscopic surgery (VATS); uniportal; lung cancer; modified

Submitted Dec 05, 2015. Accepted for publication Mar 02, 2016.

doi: 10.21037/acs.2016.03.09

View this article at: http://dx.doi.org/10.21037/acs.2016.03.09

\section{Introduction}

\section{Perspectives}

Video-assisted thoracoscopic surgery (VATS) for anatomic lung resections was first described in 1992 for a lobectomy in the surgical treatment of lung cancer $(1,2)$. VATS has been increasing in popularity over the last two decades in thoracic surgery practices worldwide $(3,4)$. The procedure depends on dissection of pulmonary arteries, veins and bronchi (main, lobar and segmental) individually without rib spreading and performing anatomical resection together with mediastinal lymph node dissection by using specifically designed thoracoscopic surgical instruments (3-6). All the surgical steps are performed with the aid of visualization via a video monitor(s) facilitated by thoracoscopic devices (3-6).

VATS lobectomy has been utilized at Duke University since 1999 and the safety and efficacy of the 2-port technique have been demonstrated (3). There have been studies showing low morbidity and low conversion rates to open thoracotomy for VATS (4-6). VATS has been proven to be beneficial for patients as it is associated with less postoperative pain, shorter hospital stay and lower rates of postoperative complications $(3,7,8)$. On a biochemical level, the benefits for patients undergoing VATS compared to conventional thoracotomy include a lower acute phase inflammatory response and lower release of interleukins and $\mathrm{C}$ reactive protein, with better preservation of the immune response $(5,9,10)$.

Technically, VATS lobectomy has been performed via 2 to 4 incisions in different centers $(4,11)$. Recently, the concept of the uniportal approach-one incision in one interspace-has been utilized, starting with minor and intermediate complexity cases such as sympathectomy, mediastinal and pleural biopsies and wedge resections. Rocco et al. reported the first uniportal VATS lung resection in 2004 (12). Gonzalez-Rivas published the first uniportal VATS lobectomy for an early stage lung cancer in 2011 (13).In recent years, more complex resections including segmentectomy, pneumonectomy, sleeve and double sleeve lung resections via this uniportal approach have been reported (14-16).

Potential disadvantages of the uniportal approach include more difficulty in teaching the operation to trainees, limited visualization, an unstable camera platform with instruments competing for space throughout the entire procedure, the chest tube exiting from the access incision and suboptimal ergonomics for the assistant and camera navigator. At Duke, the VATS approach for pulmonary resections had previously been via two incisions: an access incision of 3-4 $\mathrm{cm}$ anteriorly in the fifth intercostal space and a camera 


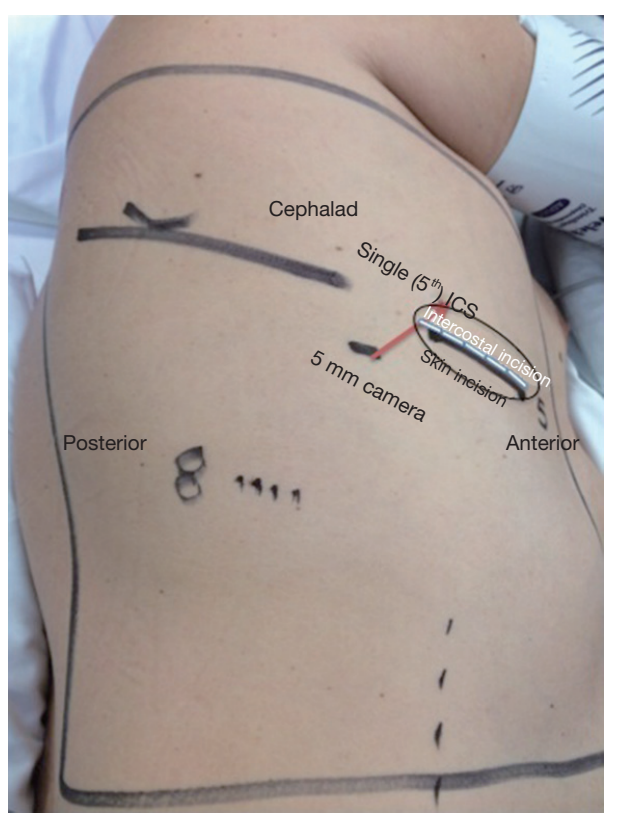

Figure 1 The labeling of the utility access incision on the $5^{\text {th }}$ intercostal space. The skin incision (black) is extended $1 \mathrm{~cm}$ anteriorly and posteriorly as an intercostal incision (white) to use the one intercostal space more efficiently. A $5 \mathrm{~mm}$ camera is placed using a separate incision below the utility access incision.

port of $10 \mathrm{~mm}$ in the seventh or eighth space $(4,8)$. We have adapted our 2-port approach experience and formed a new 'modified uniportal VATS' approach (8).

\section{Technique}

The preoperative set-up in the operating room (OR) for the modified uniportal approach is the same as for our classical VATS approach $(4,8)$. The patient is intubated selectively with either a dual lumen intubation tube or endobronchial blocker. The patient is positioned in a full lateral decubitus position and the operating side will be up with slight flexion of the table at the level of the middle of the chest. This allows slight splaying of the ribs and improves the exposure of the intercostal space in the absence of rib spreading.

The utility access incision is made at the level of the fifth intercostal space, over the anterior axillary line and no longer than 4-5 cm. Deep in the intercostal muscle layer in the involved intercostal space, we extend the incision $1 \mathrm{~cm}$ both anteriorly and posteriorly for better exposure for instrument positioning and easier retrieval of the specimen. Adjacent and posteroinferior to the access incision, a small $5-\mathrm{mm}$ 'camera port incision' is made and a trocar is placed
(Figure 1). We use a 5-mm 30-degree angled camera through the trocar. An assistant designated to operate the camera is positioned posterior to the patient. The operating surgeon stands anterior to the patient, beside the thoracic surgery trainee. As the camera navigator stands at the back of the patient, there is no difficulty in coordination of movement with the operating team. The access incision is only used for thoracoscopic surgical instrumentation including stapling devices (Figure 2).

The overall main steps in the procedure replicate our standard two-incision VATS resection. Hilar dissection is carried out through the utility incision. We begin anteriorly by dissecting the pulmonary vessels and bronchi and continue posteriorly. We are using our pre-existing VATS facilities including the surgical instruments and camera devices. Endoscopic linear and vascular stapling devices are used for individual vessel and bronchial ligation as in all VATS resections. A specimen bag is used for retrieval of the specimen through the fifth intercostal space. This avoids potential seeding of tumor cells into the access incision. Systematic mediastinal lymph node dissection and excision is done routinely for every lung cancer patient before completing the surgery. We perform 5 levels of thoracoscopic intercostal nerve blockage by using bupivacaine under direct vision. We place a $24 \mathrm{~F}$ chest tube for drainage through the camera incision. The position of the chest tube is checked by a camera placed in the access incision before closure. The chest tube is fixed with a single stitch to be tied when the tube is removed. The anterior incision is then sutured subcutaneously with absorbable material.

\section{Discussion}

\section{Pros}

In our modified uniportal VATS technique, we are using a single intercostal space for both camera and utility access. The commonly used fifth intercostal space is extended longer than the skin incision deep in the intercostal level both anteriorly and posteriorly. This helps to place the $5 \mathrm{~mm}$ thoracoscope, which has the skin incision posteriorly and is inferiorly located to the utility incision. Also, this extension decreases the tension and avoids uncontrolled pressure during removal of the specimen. The camera incision is used for placing a $24 \mathrm{~F}$ chest tube at the end of surgery. The need for placing the tube through the utility incision is no longer necessary.

With this distribution, the camera navigator stands 

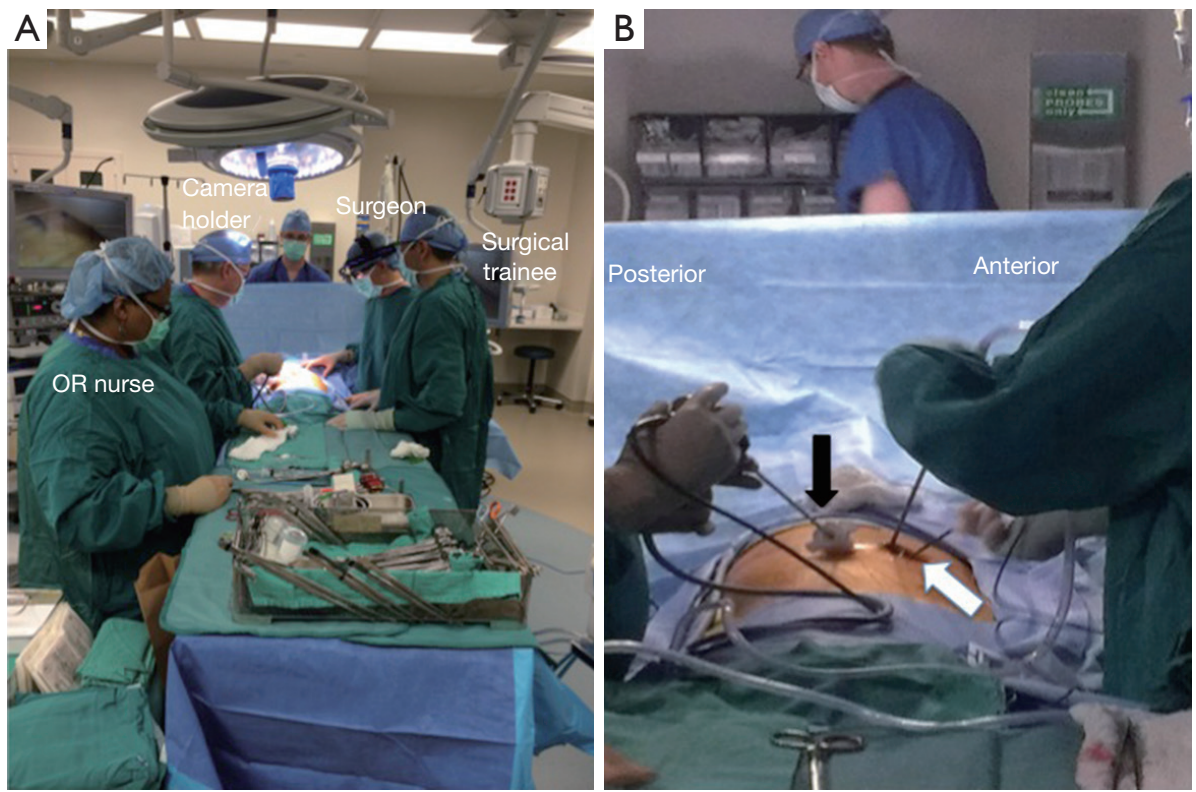

Figure 2 A $5 \mathrm{~mm}$ thoracoscope (black arrow) placed through the separate incision but using the same intercostal space as the access incision. The surgical instrumentation (white arrow) placed through the access incision with a better distribution without competing with the camera (adapted from Figure 2 of Reference 8).

posterior to the patient, which is physically comfortable and where it is easier to operate the camera without competing with the operating team and the surgical instrumentation. The thoracic surgeon and the surgical trainee (resident or fellow) stand anterior to the patient. This creates a free area for surgical manipulations if the thoracic surgeon is operating solo. Moreover, if there is a surgical trainee as mentioned, the surgeon in charge could easily supervise, guide and control the trainee. This ability to educate properly is important for increasing the use of VATS with well-educated new generations of thoracic surgeons for the future of thoracic surgery. Furthermore, by using our technique, resections of the lower lobes are easier in that there is less need to staple from a medial port.

\section{Cons}

Use of this technique requires the use of a $5 \mathrm{~mm} 30$ degree angled camera. As the instrument is thin and fragile, the camera navigator needs to exercise caution to avoid contact with the surgical instrumentation and avoid any damage to the thoracoscope or impede the maneuvers of the surgeon. Viewing some deep areas around the diaphragm could sometimes be troublesome. Although the camera port is small, the use of a separate $5 \mathrm{~mm}$ incision for the passage of the camera results in an additional scar formation in these patients.

\section{Conclusions}

VATS has been proven to be safe and beneficial compared to open thoracotomy in lung cancer patients undergoing surgical anatomic resection $(3,17)$. Uniportal VATS has been demonstrated to be safe for lung resections including increasingly complex cases. The discussion around benefits of VATS compared to conventional thoracotomy has shifted to consider conventional VATS (multiport) compared to uniportal VATS. Recent studies have demonstrated no differences in operating time, duration of patient-controlled analgesia, chest tube duration or hospital length of stay $(18,19)$. The uniportal VATS approach seems to offer improved pain scores but current evidence reveals no difference in most postoperative outcomes comparing uniportal to multiportal VATS $(20,21)$. This provides reasonable grounds to limit the procedure to one intercostal space in selected cases (8). In addition to our routine dual port VATS, we are performing modified uniportal VATS in selected patients.

The modified uniportal technique described herein retains the potential advantage of working in one interspace, while limiting the potential disadvantages of the unstable 
camera platform, suboptimal ergonomics, limited posterior visualization and the chest tube position in the access incision. Development of visualization (camera technology) and surgical instrumentation would make uniportal VATS more efficient and easier to adopt. There is still need for scientific evidence to prove the hypothetical advantages of modified uniportal and classical uniportal VATS over the traditional VATS approaches.

\section{Acknowledgements}

None.

\section{Footnote}

Conflicts of Interest: The authors have no conflicts of interest to declare.

\section{References}

1. Landreneau RJ, Mack MJ, Hazelrigg SR, et al. Video-assisted thoracic surgery: basic technical concepts and intercostal approach strategies. Ann Thorac Surg 1992;54:800-7.

2. Roviaro G, Rebuffat C, Varoli F, et al. Videoendoscopic pulmonary lobectomy for cancer. Surg Laparosc Endosc 1992;2:244-7.

3. Onaitis MW, Petersen RP, Balderson SS, et al. Thoracoscopic lobectomy is a safe and versatile procedure: experience with 500 consecutive patients. Ann Surg 2006;244:420-5.

4. Burfeind WR, D'Amico TA. Thoracoscopic lobectomy. Oper Tech Thorac Cardiovasc Surg 2004;9:98-114.

5. Gonzalez-Rivas D. VATS lobectomy: surgical evolution from conventional VATS to uniportal approach. ScientificWorldJournal 2012;2012:780842.

6. McKenna RJ Jr, Houck W, Fuller CB. Video-assisted thoracic surgery lobectomy: experience with 1,100 cases. Ann Thorac Surg 2006;81:421-5; discussion 425-6.

7. Villamizar NR, Darrabie MD, Burfeind WR, et al. Thoracoscopic lobectomy is associated with lower morbidity compared with thoracotomy. J Thorac Cardiovasc Surg 2009;138:419-25.

8. Kara HV, Balderson SS, D'Amico TA. Modified uniportal video-assisted thoracoscopic lobectomy: Duke approach. Ann Thorac Surg 2014;98:2239-41.

9. Sugi K, Kaneda Y, Esato K. Video-assisted thoracoscopic lobectomy reduces cytokine production more than conventional open lobectomy. Jpn J Thorac Cardiovasc Surg 2000;48:161-5.
10. Yim AP, Wan S, Lee TW, et al. VATS lobectomy reduces cytokine responses compared with conventional surgery. Ann Thorac Surg 2000;70:243-7.

11. Ng CS, Gonzalez-Rivas D, D'Amico TA, et al. Uniportal VATS-a new era in lung cancer surgery. J Thorac Dis 2015;7:1489-91.

12. Rocco G, Martucci N, La Manna C, et al. Ten-year experience on 644 patients undergoing single-port (uniportal) video-assisted thoracoscopic surgery. Ann Thorac Surg 2013;96:434-8.

13. Gonzalez-Rivas D, de la Torre M, Fernandez R, et al. Singleport video-assisted thoracoscopic left upper lobectomy. Interact Cardiovasc Thorac Surg 2011;13:539-41.

14. Gonzalez-Rivas D, Fieira E, Delgado M, et al. Uniportal video-assisted thoracoscopic sleeve lobectomy and other complex resections. J Thorac Dis 2014;6:S674-81.

15. Gonzalez-Rivas D. Single incision video-assisted thoracoscopic anatomic segmentectomy. Ann Cardiothorac Surg 2014;3:204-7.

16. Gonzalez-Rivas D, Delgado M, Fieira E, et al. Uniportal video-assisted thoracoscopic pneumonectomy. J Thorac Dis 2013;5 Suppl 3:S246-52.

17. Berry MF, D'Amico TA, Onaitis MW, et al. Thoracoscopic approach to lobectomy for lung cancer does not compromise oncologic efficacy. Ann Thorac Surg 2014;98:197-202.

18. McElnay PJ, Molyneux M, Krishnadas R, et al. Pain and recovery are comparable after either uniportal or multiport video-assisted thoracoscopic lobectomy: an observation study. Eur J Cardiothorac Surg 2015;47:912-5.

19. Chung JH, Choi YS, Cho JH, et al. Uniportal videoassisted thoracoscopic lobectomy: an alternative to conventional thoracoscopic lobectomy in lung cancer surgery? Interact Cardiovasc Thorac Surg 2015;20:813-9.

20. Akter F, Routledge T, Toufektzian L, et al. In minor and major thoracic procedures is uniport superior to multiport video-assisted thoracoscopic surgery? Interact Cardiovasc Thorac Surg 2015;20:550-5.

21. Jimenez MF. Uniportal versus standard video-assisted thoracoscopic surgery for lung lobectomy: changing the standards requires scientific evidence. Eur J Cardiothorac Surg 2015;47:916.

Cite this article as: Kara HV, Balderson SS, D'Amico TA. Modified uniportal video-assisted thoracoscopic surgery (VATS). Ann Cardiothorac Surg 2016;5(2):123-126. doi: 10.21037/acs.2016.03.09 